\title{
EDITORIAL \\ Mapping at rest: translating resting-state functional MRI to clinical practice
}

\author{
Jarod L. Roland, MD, and Matthew D. Smyth, MD \\ Department of Neurological Surgery, Washington University, St. Louis Children's Hospital, St. Louis, Missouri
}

$\mathrm{R}$ ESTING-STATE functional MRI (rs-fMRI) is a rapidly maturing methodology for noninvasive human brain mapping. Recent years have shown an exponential growth in rs-fMRI research that continues to demonstrate its utility across a wide range of clinical and basic science applications..$^{13}$ It has proven immensely useful in the study of cognitive neuroscience and is being translated to the clinical setting for diagnostic testing and preoperative evaluation. A significant portion of this growth has been developing applications in neurosurgery for surgical planning.

Neuroscientists have contributed a vast effort to understanding the neurophysiological basis of resting-state functional connectivity. The underlying substrate in a functional MRI (fMRI) study is the blood oxygen leveldependent (BOLD) signal, which reflects changes in cerebral blood flow that occur in response to neural activity. Therefore, the BOLD signal is an indirect measure of brain function. Understanding the complex dynamics of the BOLD signal is not straightforward, and it is only on the shoulders of numerous neuroimaging researchers that we are able to analyze and interpret these data. The pioneering applications of BOLD signal analysis were realized with block-design paradigms in which patients perform motor, speech, or other tasks alternating with rest epochs to systematically map functional areas involved with performing a single task. This type of task-based fMRI continues to serve a valuable role in clinical neurosurgery but may soon be supplemented, or supplanted, by rs-fMRI. The fundamental advantages of rs-fMRI are that task performance is not necessary and multiple functional brain networks may be mapped simultaneously.

Neurosurgeon scientists have maintained a prominent role in the history of human brain mapping. Wilder Penfield is one such prototypical neurosurgeon who is largely credited with developing the modern practice of direct cor- tical stimulation (DCS) mapping. The passive counterpart to DCS is electrocorticography (ECoG) for recording the electrophysiological signals directly from the surface of the brain. The combination of DCS and ECoG has proven to be invaluable in clinical neurosurgery. ECoG analysis has evolved over time with advanced methodologies afforded by greater computing power. For example, digital signal processing techniques applied to invasive electrophysiology allow for automated and real-time functional mapping in neurosurgical patients. ${ }^{12}$ This type of analysis can be performed under a variety of conditions, such as while a patient is performing tasks ${ }^{8}$ or at rest, ${ }^{3}$ as well as in the operating room ${ }^{10}$ or at the bedside..$^{15}$ However, while ECoG affords a number of advantages, such as high temporal and spatial resolution, it remains inherently invasive and restricted in its coverage to the area of brain accessible via craniotomy. For this reason, it is highly advantageous to pursue noninvasive techniques for complementary means of preoperative brain mapping. This is where functional MRI excels, and rs-fMRI becomes a logical progression in the art of brain mapping.

Early rs-fMRI work focused on group data to identify the commonalities among brain networks when averaged over multiple individuals. Recent advances have focused a spotlight on the ability to study networks at the individual level with greater accuracy. ${ }^{4,6}$ Such individual mapping is a fundamental necessity for the successful application in a clinical setting. Another result important for successful clinical application was reported by Gratton et al., who showed that the patterns of a resting-state network (RSN) captured by r-fMRI are stable within the person being studied when measured across multiple task states. ${ }^{5}$ This supports the ability to reliably identify specific areas belonging to functionally defined networks and that those areas are stable within a person, despite significant spatial variability between individuals. Together, these and other 
studies suggest that rs-fMRI on an individual level, rather than a group level, is not only technically feasible but also reliable and relevant to the clinical setting.

Individualized brain mapping is the quintessential utility of functional connectivity that is directly applicable to clinical neurosurgery. Advancing that realization is the subject of a paper by Zacà et al. in the current issue of the Journal of Neurosurgery. ${ }^{16}$ The authors demonstrate the application of an automated method for identifying RSNs in 6 patients prior to neurosurgical resection of intraparenchymal lesions. An automated method such as theirs is useful, and arguably necessary, for the widespread adoption of rs-fMRI in neurosurgical practice.

While there exist myriad techniques for analyzing rs-fMRI, the 2 most common methods are seed-based analysis and independent component analysis (ICA). Both methods are widely used and have their respective advantages and disadvantages. Seed-based analysis uses predefined locations for placing a "seed" from which a reference signal is measured and then compared with those signals from all other areas of the brain. Functionally related areas have a strong temporal correlation in their measured signals, which allows for identification of a socalled resting-state network. This was the technique used by Biswal et al. in the foundational study demonstrating the ability to map the sensorimotor system from an individual at rest. ${ }^{2}$ The disadvantage of a seed-based technique is that it depends on the correct placement of a seed to identify the relevant functional network. Disruptions of anatomy, such as from the mass effect of an intracranial tumor, may result in inaccurate seed placement and erroneous RSN maps. An alternative solution is ICA, which is a blind source separation technique that separates the signals into maximally independent spatial sources. ${ }^{1}$ It is data driven and not dependent on an a priori-defined seed placement. The resultant components represent RSNs as well as noise and nuisance signals. Such an approach is attractive for less dependence on prior information based on group-averaged data, but it is reliant on an expert reviewer to interpret the resultant components and assign them to a corresponding RSN.

Zacà et al. build on the ICA method with the addition of an automated step for matching components to a predefined RSN template map. ${ }^{16}$ This effectively emulates the process of an expert reviewer assigning an RSN to each component but does so systematically using a goodnessof-fit test. The authors introduce a software package named resting-state neurosurgical mapping, or ReStNeuMap, that implements this step, along with routine preprocessing, in a turn-key pipeline intended for use in presurgical planning. The software is readily available for download via their laboratory website. Providing a freely available software solution, such as the authors describe, for other neurosurgeons to implement is a crucial step in advancing the widespread adoption of rs-fMRI mapping in clinical practice. We congratulate the authors on this achievement and are eager to see further work on this topic.

Other groups have reported similar approaches to adapting rs-fMRI for surgical planning. Lu et al. described a comparable method for automated matching of ICA components to an RSN template for mapping the language network in brain tumor patients. ${ }^{7}$ Similarities between the studies by Zacà and Lu and their colleagues include examining varying numbers of components in the ICA, matching components to a template map in an automated manner, and comparing results to invasive stimulation mapping for verification. An earlier study by Tie et al. also reported a method for matching individual ICA results to a template language network map in patients preoperatively. ${ }^{14}$ Their method was semiautomated and compared to task-based fMRI data in healthy individuals. The results described by $\mathrm{Lu}$ et al. and Tie et al. are limited to identifying the language network. Hacker et al. described training a machine learning algorithm, the multilayer perceptron, for automated mapping of 7 canonical RSNs. ${ }^{6}$ This method was then compared with stimulation mapping for motor and language networks in tumor and epilepsy patients ${ }^{9}$ and also reported in a series of pediatric neurosurgical patients, ${ }^{11}$ where the requirement of task performance in functional mapping may be particularly challenging.

The work by Zacà et al. and related reports by other groups make a compelling proposal for the future of rsfMRI in clinical neurosurgery. ${ }^{16}$ Future work along similar lines to further validate methodologies and disseminate tools to the neurosurgical masses is eagerly awaited. While there is plenty of work to be done, we expect that neurosurgeons and basic neuroscientists will continue to work together to advance this effort and successfully translate rs-fMRI to a common clinical application.

https://thejns.org/doi/abs/10.3171/2018.5.JNS181057

\section{References}

1. Beckmann CF, DeLuca M, Devlin JT, Smith SM: Investigations into resting-state connectivity using independent component analysis. Philos Trans R Soc London Ser B Biol Sci 360:1001-1013, 2005

2. Biswal B, Yetkin FZ, Haughton VM, Hyde JS: Functional connectivity in the motor cortex of resting human brain using echo-planar MRI. Magn Reson Med 34:537-541, 1995

3. Breshears JD, Gaona CM, Roland JL, Sharma M, Bundy DT, Shimony JS, et al: Mapping sensorimotor cortex with slow cortical potential resting-state networks while awake and under anesthesia. Neurosurgery 71:305-316, 2012

4. Gordon EM, Laumann TO, Gilmore AW, Newbold DJ, Greene DJ, Berg JJ, et al: Precision functional mapping of individual human brains. Neuron 95:791-807, 807.e1-807. e7, 2017

5. Gratton C, Laumann TO, Nielsen AN, Greene DJ, Gordon EM, Gilmore AW, et al: Functional brain networks are dominated by stable group and individual factors, not cognitive or daily variation. Neuron 98:439-452, 452.e1-452.e5, 2018

6. Hacker CD, Laumann TO, Szrama NP, Baldassarre A, Snyder AZ, Leuthardt EC, et al: Resting state network estimation in individual subjects. Neuroimage 82:616-633, 2013

7. Lu J, Zhang H, Hameed NUF, Zhang J, Yuan S, Qiu T, et al: An automated method for identifying an independent component analysis-based language-related resting-state network in brain tumor subjects for surgical planning. Sci Rep 7:13769, 2017

8. Miller KJ, Leuthardt EC, Schalk G, Rao RPN, Anderson NR, Moran DW, et al: Spectral changes in cortical surface potentials during motor movement. J Neurosci 27:2424-2432, 2007 
9. Mitchell TJ, Hacker CD, Breshears JD, Szrama NP, Sharma M, Bundy DT, et al: A novel data-driven approach to preoperative mapping of functional cortex using resting-state functional magnetic resonance imaging. Neurosurgery 73:969-983, 2013

10. Roland J, Brunner P, Johnston J, Schalk G, Leuthardt EC: Passive real-time identification of speech and motor cortex during an awake craniotomy. Epilepsy Behav 18:123-128, 2010

11. Roland JL, Griffin N, Hacker CD, Vellimana AK, Akbari $\mathrm{SH}$, Shimony JS, et al: Resting-state functional magnetic resonance imaging for surgical planning in pediatric patients: a preliminary experience. J Neurosurg Pediatr 20:583-590, 2017

12. Schalk G, Leuthardt EC, Brunner P, Ojemann JG, Gerhardt LA, Wolpaw JR: Real-time detection of event-related brain activity. Neuroimage 43:245-249, 2008

13. Snyder AZ: Intrinsic brain activity and resting state networks, in Pfaff DW, Volkow ND (eds): Neuroscience in the 21st Century. New York: Springer, 2015, pp 1-52

14. Tie Y, Rigolo L, Norton IH, Huang RY, Wu W, Orringer D, et al: Defining language networks from resting-state fMRI for surgical planning - a feasibility study. Hum Brain Mapp 35:1018-1030, 2014

15. Wu M, Wisneski K, Schalk G, Sharma M, Roland J, Breshears J, et al: Electrocorticographic frequency alteration mapping for extraoperative localization of speech cortex. Neurosurgery 66:E407-E409, 2010

16. Zacà D, Jovicich J, Corsini F, Rozzanigo U, Chioffi F, Sarubbo S: ReStNeuMap: a tool for automatic extraction of resting-state fMRI networks in neurosurgical practice. J Neurosurg [epub ahead of print October 26, 2018. DOI: 10.3171/2018.4.JNS18474]

\section{Disclosures}

The authors report no conflict of interest.

\section{Correspondence}

Matthew D. Smyth: mdsmyth@wustl.edu.

\section{INCLUDE WHEN CITING}

Published online October 26, 2018; DOI: 10.3171/2018.5.JNS181057. 